\title{
Co-encapsulation of curcumin and doxorubicin in albumin nanoparticles blocks the adaptive treatment tolerance of cancer cells
}

\author{
Seyed Mohammad Motevalli, ${ }^{1,2}$ Ahmed Shaker Eltahan ${ }^{1,2}$, Lu Liu ${ }^{1,2,3}$, \\ Andrea Magrini $^{4,5}$, Nicola Rosato ${ }^{3,5}$, Weisheng Guo ${ }^{1}$, Massimo Bottini ${ }^{1,3,5,6}$, \\ Xing-Jie Liang ${ }^{1,2} \bowtie$
}

1 CAS Key Laboratory for Biomedical Effects of Nanomaterials and Nanosafety, CAS Center for Excellence in Nanoscience, National Center for Nanoscience and Technology of China, Beijing 100190, China

2 University of Chinese Academy of Sciences, Beijing 100049, China

3 Department of Experimental Medicine, University of Rome Tor Vergata, Via Montpellier 1, 00133 Rome, Italy

4 Department of Biomedicine and Prevention, University of Rome Tor Vergata, Via Montpellier 1, 00133 Rome, Italy

5 Nanoscience \& Nanotechnology \& Innovative Instrumentation (NAST) Centre, University of Rome Tor Vergata, 00133 Rome, Italy

6 Inflammatory and Infectious Disease Center, Sanford-Burnham Medical Research Institute, 10901 North Torrey Pines Road, La Jolla, CA 92037, USA

Received: 17 May 2018 / Accepted: 27 August 2018 / Published online: 11 January 2019

\begin{abstract}
The adaptive treatment tolerance (ATT) of cancer cells is the main encumbrance to cancer chemotherapy. A potential solution to this problem is to treat cancer cells with multiple drugs using nanoparticles (NPs). In this study, we tested the co-administration of curcumin (Cur) and doxorubicin (Dox) to MCF-7 resistant breast cancer cells to block the ATT and elicit efficient cell killing. Drugs were co-administered to cells both sequentially and simultaneously. Sequential drug co-administration was carried out by pre-treating the cells with albumin nanoparticles (ANPs) loaded with Cur (Cur@ANPs) followed by treatment with Dox-loaded ANPs (Dox@ANPs). Simultaneous drug co-administration was carried out by treating the cells with ANPs loaded with both the drugs (Cur/Dox@ANPs). We found that the simultaneous drug co-administration led to a greater intra-cellular accumulation of Dox and cell killing with respect to the sequential drug co-administration. However, the simultaneous drug co-administration led to a lower intracellular accumulation of Cur with respect to the sequential drug co-administration. We showed that this result was due to the aggregation and entrapment of Cur in the lysosomes as soon as it was released from Cur@ANPs, a phenomenon called lysosomotropism. In contrast, the simultaneous release of Dox and Cur from Cur/Dox@ANPs into the lysosomes led to lysosomal pH elevation, which, in turn, avoided Cur aggregation, led to lysosome swelling and drug release in the cytosol, and finally provoked efficient cell killing. Our study shed the light on the molecular processes driving the therapeutic effects of anti-cancer drugs co-administered to cancer cells in different manners.
\end{abstract}

Keywords Albumin nanoparticles, Doxorubicin, Curcumin, P-glycoprotein, Lysosomal pH

Seyed Mohammad Motevalli and Ahmed Shaker Eltahan have contributed equally to this work.

$\bowtie$ Correspondence: massimo.bottini@uniroma2.it (M. Bottini), liangxj@nanoctr.cn (X.-J. Liang)

\section{INTRODUCTION}

One of the main challenges in the field of cancer therapy is the poor sensitivity to anti-cancer drugs developed by 
cancer cells after receiving the therapeutic treatment for a prolonged time, a phenomenon named adaptive treatment tolerance (ATT) (Zhou et al. 2015). ATT could be caused by several processes, including the difficulty of drugs to pass through the plasma membrane and/or escape from the lysosomal compartments of cancer cells or the transport of drugs out of cancer cells due to the activation of multidrug resistance (MDR)-associated proteins (Housman et al. 2014; Logan et al. 2014; Tarragó-Trani and Storrie 2007; Zhitomirsky and Assaraf 2016). One potential approach used to address the ATT is the co-administration of anti-cancer drugs to cancer cells (Wang et al. 2013a). Drugs can be co-administered to cells in two different manners, i.e., sequentially and simultaneously. In the sequential manner of co-administration, cells are pre-treated with a chemo-sensitizer, followed by the administration of a chemo-drug (Neville-Webbe et al. 2005). Pre-treatment of cancer cells with chemosensitizers significantly increases the efficacy of chemodrugs. Pre-treatment with Erlotinib inhibited the epidermal growth factor receptor (EGFR) and increased the therapeutic efficacy of doxorubicin (Dox) in a subset of triple-negative breast cancers (Lee et al. 2012). However, the sequential co-administration of drugs to cancer cells is not always the best option (Neek et al. 2018). Ducreux et al. have shown that the simultaneous co-administration of drugs led to more cytotoxic effects on advanced, resectable colorectal cancer compared to the sequential drug co-administration (Ducreux et al. 2011). One potential explanation for these differences is that anti-cancer drugs often display poor solubility in aqueous environments due to their hydrophobic nature. To address this problem, anti-cancer drugs have been encapsulated into nanoparticles (NPs). NPs can improve the therapeutic efficacy of drugs by increasing their halflife in the blood circulation, facilitating their cell penetration and intra-cellular distribution, and decreasing their side effects on normal tissues and cells (Bronze-Uhle et al. 2017; She et al. 2013; Yan et al. 2016).

Among the NPs used for cancer treatment, albuminbased NPs (ANPs) have been widely used as drug carriers due to their biocompatibility, lack of immunogenicity and toxicity, and great storage stability (Kratz 2008; Li et al. 2015; Lohcharoenkal et al. 2014). ANPs can be prepared in various range of sizes by means of straightforward, inexpensive, and easily controlled preparation procedures (Céspedes et al. 2014). Importantly, ANPs can be loaded with both hydrophilic and hydrophobic cargo (Cheema et al. 2009; Lin et al. 2011) and display a favorable pharmacokinetic profile due to their ability to accumulate in tumors by exploiting the enhanced permeability and retention (EPR) effect (Abbasi et al. 2012) and/or by triggering transcytosis in the endothelial cells by interacting with the albondin/glycoprotein 60 (Azizi et al. 2017). ANPs have been already approved for treatment of different cancers. Paclitaxel-loaded ANPs (Abraxane) have been approved for the treatment of breast cancer and clinical studies on docetaxel-loaded ANPs and are in progress for the treatment of several types of tumors.

Dox has been a widely used anti-cancer anthracycline but is suffering from serious side effects, including a dosedependent and cumulative life-threatening cardiotoxicity and the development of MDR (Carvalho et al. 2014). These shortcomings have been tackled by entrapping Dox into NPs and using chemo-sensitizers (Deepa et al. 2014). In this study, we investigated the co-treatment of MCF-7 resistant breast cancer cells with Dox and the chemosensitizer curcumin (Cur). Cur is a natural compound that inhibits the MDR protein family-member P-glycoprotein (P-gp) and blocks the transport of anti-cancer drugs out of cancer cells (Hsieh et al. 2014; Neerati et al. 2013; Peng et al. 2018; Sahu et al. 2016; Umsumarng et al. 2015; Vinod et al. 2013; Wang et al. 2016; Zhang et al. 2016). Both the drugs were encapsulated into ANPs and co-administered to cells both sequentially and simultaneously. Sequential drug co-administration was carried out by pre-treating the cells with ANPs loaded with Cur (Cur@ANPs) followed by treatment with ANPs loaded with Dox (Dox@ANPs). Simultaneous drug co-administration was carried out by treating the cells with ANPs loaded with both the drugs (Cur/Dox@ANPs). We found that the simultaneous drug co-administration led to a greater intra-cellular accumulation of Dox and cell killing with respect to the sequential drug co-administration. However, the simultaneous drug co-administration led to a lower intra-cellular accumulation of Cur with respect to the sequential drug co-administration. We showed that this result was due to the aggregation and entrapment of Cur in the lysosomes as soon as it was released from Cur@ANPs (lysosomotropism). On the contrary, the simultaneous release of Dox and Cur from Cur/Dox@ANPs into the lysosomes led to lysosomal $\mathrm{pH}$ elevation, which, in turn, avoided Cur aggregation, led to lysosome swelling and the release of the drugs in the cytosol, and finally provoked efficient cell killing. Our study showed the importance of tuning the manner of co-administration of chemo-drugs and chemosensitizers in order to maximize the therapeutic outcome.

\section{RESULTS AND DISCUSSION}

\section{Preparation and characterization of albumin nanoparticles loaded with Cur and/or Dox}

Bovine serum albumin (BSA) nanoparticles (ANPs) loaded with Cur (Cur@ANPs), Dox (Dox@ANPs), or both 
of them (Cur/Dox@ANPs) were prepared by mixing the $\operatorname{drug}(\mathrm{s})$ to a solution of BSA. Due to their hydrophobic nature, Cur and Dox will be forced to aggregate in an aqueous environment and interact with the hydrophobic regions of the BSA forming micelle-like structures, which will be stabilized by cross-linking the surface amino groups of BSA with glutaraldehyde (Bronze-Uhle et al. 2017; Gong et al. 2017; Ma et al. 2016). First, we characterized the physical and chemical properties of the NPs by transmission electron microscopy (TEM) and dynamic light scattering (DLS). TEM images showed that all the three types of NPs had a spheroidal shape with sizes ranging from 20 to $30 \mathrm{~nm}$ and were individually dispersed in a dehydrated state (Fig. 1A and Table 1). DLS measurements showed that the NPs had an average hydrodynamic diameter in the $80-100 \mathrm{~nm}$ range and a negatively charged surface in a hydrated state (Fig. 1B and Table 1). It is worth noting that the diameter of the NPs calculated by means of TEM images was smaller than that given by DLS data due to the shrinkage of the NPs during the drying of the TEM grids before imaging. All the NPs were negatively charged with the order Dox@ANPs $(-7.53 \pm 0.7 \mathrm{mV})>$ Cur/ Dox@ANPs $(-11.6 \pm 1.2 \mathrm{mV})>$ Cur@ANPs $(-18.2 \pm$ $1.3 \mathrm{mV}$ ) (Table 1). The fact that Dox@ANPs had the highest surface charge among the fabricated NPs was most likely due to the amino groups in Dox.

Next, we tested the encapsulation of Cur and/or Dox in the ANPs. The encapsulation efficacy percentage $(E E \%)$ and loading yield percentage $(L Y \%)$ were measured by means of HPLC. The $E E \%$ was greater than $85 \%$ for both Cur and Dox in all the NPs tested (Table 1). UV-Vis spectroscopy, differential scanning calorimetry (DSC), and Fourier-transform infrared (FTIR) spectroscopy were used to further test the encapsulation of Cur and/or Dox in the NPs. Free Cur and Dox and their NP counterparts exhibited an absorbance peak at 427 and $495 \mathrm{~nm}$, respectively, whereas both a mixture of free drugs and Cur/ Dox@ANPs exhibited a broad absorbance peak $\sim 430 \mathrm{~nm}$ with a shoulder at $\sim 490 \mathrm{~nm}$ (Fig. 1C). These results validated the encapsulation of a single drug or both the drugs in the BSA matrix. Additionally, the DSC spectra of the NPs did not show the melting point peak(s) of Cur and/or Dox, which suggested that the drugs were in an amorphous state upon encapsulation in the NPs (Jenita et al. 2014) (Fig. 1D). Finally, we recorded the FTIR spectra of BSA, Cur, Dox, and the fabricated NPs (Fig. 1E). The FTIR spectra of BSA showed several characteristic peaks including 2925, 1651 , and $1531 \mathrm{~cm}^{-1}$, which are the representing peaks of amid I (mainly -NH stretching vibrations), amid II (mainly $\mathrm{C}=\mathrm{O}$ stretching vibrations), and amid III (the coupling stretching vibrations of $\mathrm{C}-\mathrm{N}$ and the coupling bending vibrations of $-\mathrm{NH}$ ), respectively (Huang et al. 2010). The main FTIR peaks of Cur were found at $3506 \mathrm{~cm}^{-1}$ as a result of $-\mathrm{OH}$ stretching vibrations, $1271 \mathrm{~cm}^{-1}$ for aromatic $\mathrm{C}-0$ stretching vibrations, and $1023 \mathrm{~cm}^{-1}$ due to $\mathrm{C}-0-\mathrm{C}$ stretching vibrations (Darandale and Vavia 2013; Mohanty and Sahoo 2010; Wang et al. 2013b; Yallapu et al. 2010). The main characteristics peaks of Dox were found at $3450 \mathrm{~cm}^{-1}$ as a result of $-\mathrm{NH}_{2}$ stretching vibrations, $1410 \mathrm{~cm}^{-1}$ due to $\mathrm{C}-\mathrm{C}$ vibrations, and $1073 \mathrm{~cm}^{-1}$ from $\mathrm{C}=0$ vibrations (Jayakumar et al. 2012; Unsoy et al. 2014). The presence of the relevant peaks in each type of NP confirmed that the Cur and/or Dox were encapsulated in the BSA matrix.

Finally, we tested the release of the drugs from the NPs in an acidic buffer to mimic the conditions encountered by the NPs upon accumulation in the lysosomes. The conformational changes in the protein structure induced by the acidic conditions led to the release of both Cur and Dox from the NPs with a firstorder kinetics (Fig. 2A, B). The speed of release of both the drugs increased with the acidity of the environment; however, Cur showed a slower kinetics of release with respect to Dox. This phenomenon was explained by considering that Cur is more hydrophobic than Dox and interacts with the hydrophobic domains of BSA more strongly than Dox (Chen et al. 2015; Dreis et al. 2007; Li et al. 2008). We also assessed the effect of the shell of glutaraldehyde molecules cross-linking the amino groups on the ANPs' surface on the kinetics of drug release. We reasoned that the kinetics of drug release would decrease with increasing the density of crosslinked surface amino groups. In order to validate this hypothesis, we prepared NPs with higher densities of crosslinked surface amino groups by increasing both the glutaraldehyde: BSA molar ratio and the reaction time. As hypothesized, NPs with higher densities of crosslinked surface amino groups displayed slower kinetics of drug release (Fig. 2C-E).

\section{Cytotoxicity of albumin nanoparticles loaded with Cur and/or Dox on MCF-7 cancer cells}

MCF-7 cancer cells were chosen for our experiments because overexpressing the P-gp pump (Lin et al. 2014; $\mathrm{Lv}$ et al. 2014). Cells were divided into five groups. Three groups of cells were treated with either Cur@ANPs or Dox@ANPs or Cur/Dox@ANPs loaded with $15,25,40$, or $60 \mu \mathrm{mol} / \mathrm{L}$ of Cur and/or Dox for $24 \mathrm{~h}$. The fourth and the fifth group of cells was pre-treated with Cur@ANPs loaded with 15, 25, 40, or $60 \mu \mathrm{mol} / \mathrm{L}$ of Cur for 6 or $12 \mathrm{~h}$, respectively, and then the cells were exposed to Dox@ANPs loaded with a concentration of 
A

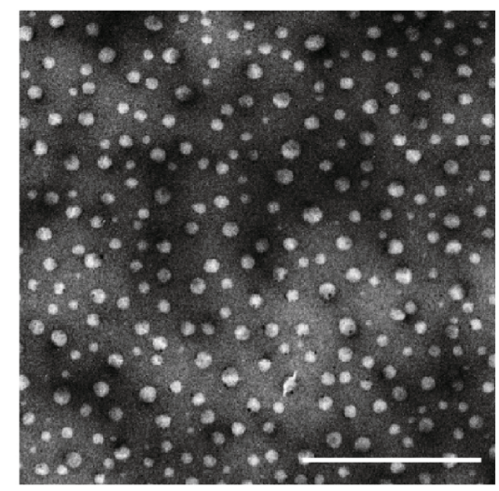

Dox@ANPs

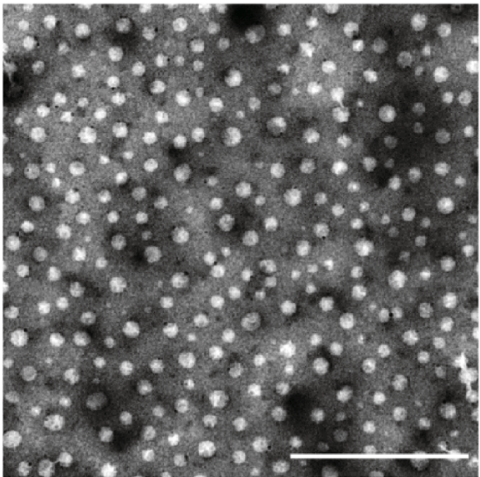

Cur/Dox@ANPs

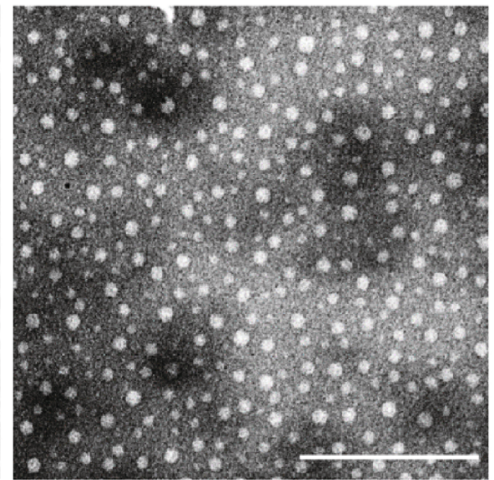

B

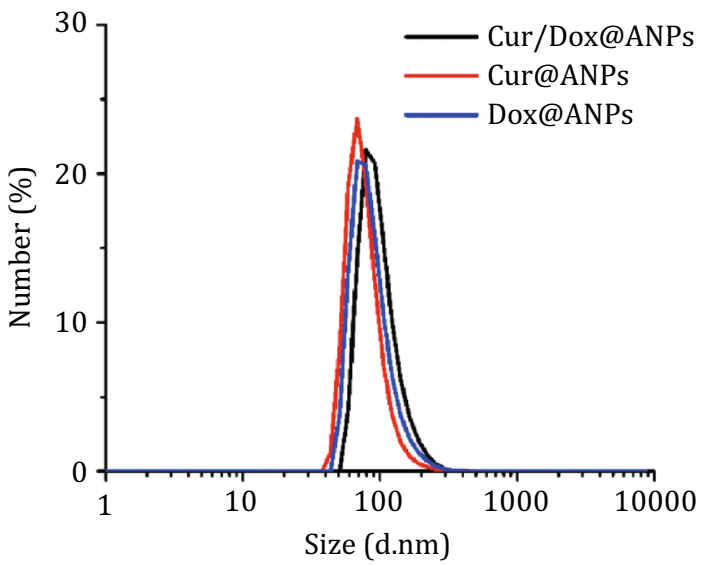

C

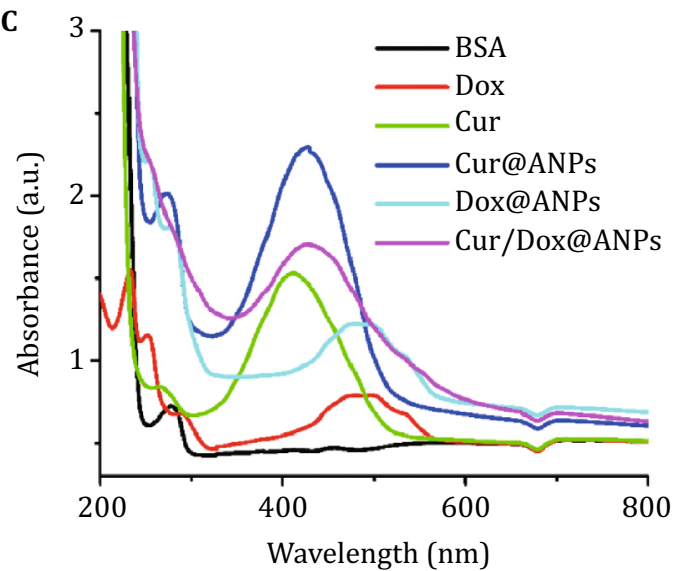

E

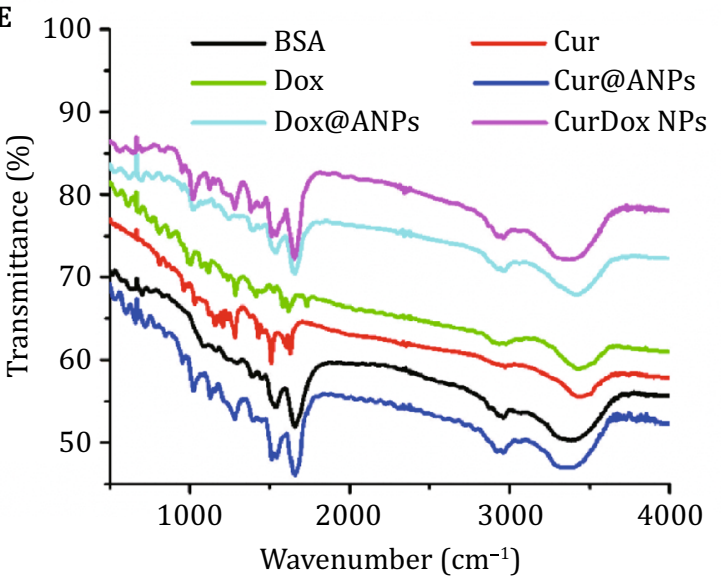

Fig. 1 Characterization of the physical and chemical properties of Cur@ANPs, Dox@ANPs, and Cur/Dox@ANPs. A TEM images of the NPs (dehydrated state, scale bars are $200 \mathrm{~nm}$ ). B DLS analyses of the NPs (hydrated state). C UV-Vis absorption spectra. D DSC spectra. E Fourier-transform infrared spectra

Dox equal to that of Cur in Cur@ANPs for an additional $24 \mathrm{~h}$. The MTT assay showed that the cell viability decreased with the concentration of the drugs independently from the type of treatment. We also found that the cells treated with Cur/Dox@ANPs exhibited a lower viability than the cells treated with either Cur@ANPs or Dox@ANPs for all the drug concentrations tested. Additionally, we found that the cells exposed to Cur/Dox@ANPs showed lower viability than the cells pre-treated with Cur@ANPs for 6 and $12 \mathrm{~h}$, for all the drug concentrations tested (Fig. 3A). We assessed if this phenomenon was due to a greater uptake of the drugs by cells treated with Cur/Dox@ANPs with respect to the cells pre-treated with Cur@ANPs by means of FACS. As expected, we found that the accumulation of Dox in MCF-7 cancer cells was the greatest 
Table 1 Physical properties of Cur@ANPs, Dox@ANPs, and Cur/Dox@ANPs

\begin{tabular}{lllllll}
\hline Nanoparticle & $\zeta(\mathrm{mV})$ & Size, TEM $(\mathrm{nm})$ & Size, DLS $(\mathrm{nm})$ & PDI & EE\% & LY\% \\
\hline Cur/Dox@ANPs & $-11.6 \pm 1.2$ & 31.4 & $99.94 \pm 8.6$ & $0.193 \pm 0.006$ & Cur: 95.46 & Cur: 9.3 \\
& & & & & Dox: 86.69 & Dox: 13.27 \\
Cur@ANPs & $-18.2 \pm 1.3$ & 22.1 & $78.84 \pm 7.3$ & $0.359 \pm 0.043$ & 98.2 & 3 \\
Dox@ANPs & $-7.53 \pm 0.7$ & 25.2 & $88.3 \pm 6$ & $0.201 \pm 0.004$ & 87.34 & 4.1 \\
\hline
\end{tabular}

$\zeta$ : zeta potential, PDI: polydispersity index

A

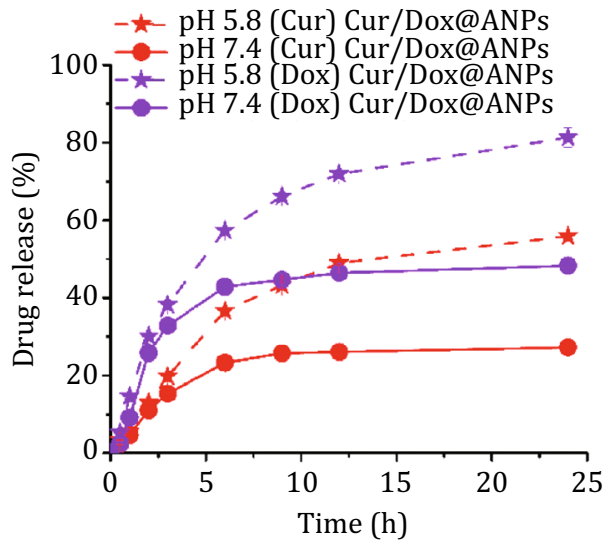

B $\quad-\star-$ pH 5.8 (Cur) Cur@ANPs

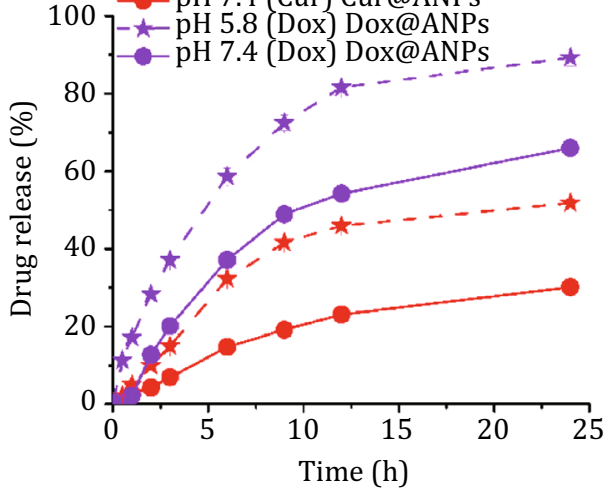

C

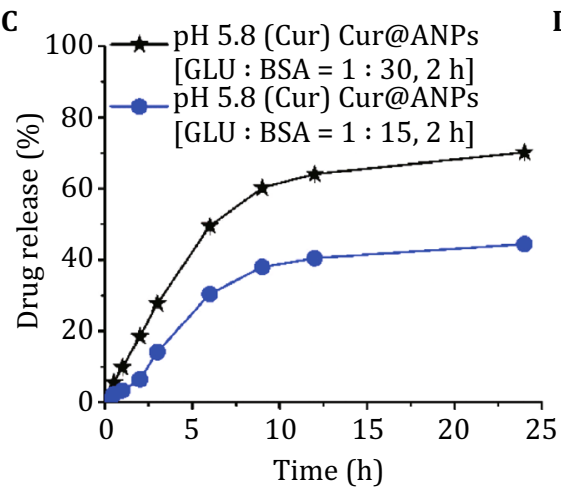

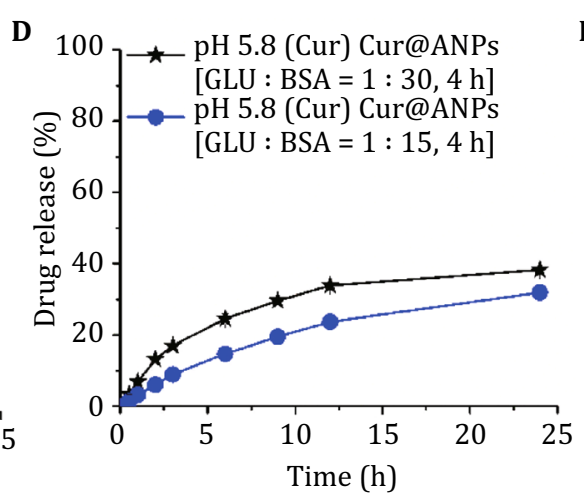

E 100 - pH 5.8 (Cur) Cur@ANPs

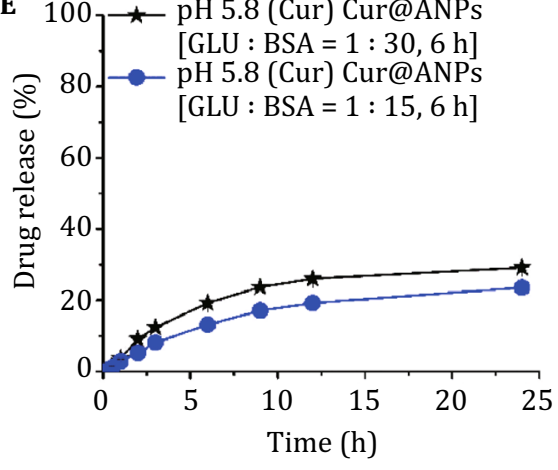

Fig. 2 Kinetic analysis of Cur and Dox release from Cur@ANPs, Dox@ANPs, and Cur/Dox@ANPs at neutral and acidic pH. A Drug release kinetics from Cur/Dox@ANPs. B Drug release kinetics from Cur@ANPs and Dox@ANPs at pH 5.8 and 7.4. C-E Kinetic analysis of Cur release from Cur@ANPs fabricated at different glutaraldehyde: BSA molar ratios and reaction times. The experiment was conducted at $\mathrm{pH} 5.8$

when they were treated with Cur/Dox@ANPs for all the drug concentrations tested (Fig. 3B). However, cells pretreated with Cur@ANPs took up more Cur than cells treated with Cur/Dox@ANPs (Fig. 3C), which was in contrast with the cell viability data. We hypothesized that the pre-treatment with Cur@ANPs did not lead to efficient cytotoxicity because Cur aggregated and remained entrapped in the lysosomes as soon as it was released from the NPs due to its weak-base nature (lysosomotropism) (Kuzu et al. 2017). In order to validate this hypothesis, we assessed the trafficking profile of Cur in cells treated with Cur/Dox@ANPs for $3 \mathrm{~h}$ and in cells pre-treated with Cur@ANPs for $1.5 \mathrm{~h}$ and then exposed to Dox@ANPs for an additional $1.5 \mathrm{~h}$ by means of confocal laser scanning microscopy (CLSM) (Burger et al. 2015). Cells pre-treated with Cur@ANPs showed a strong punctuate fluorescence arising from Cur co-localized with the lysosomes and a faint fluorescence arising from Dox (Fig. 4B), whereas cells exposed to Cur/Dox@ANPs showed diffused signals 
A

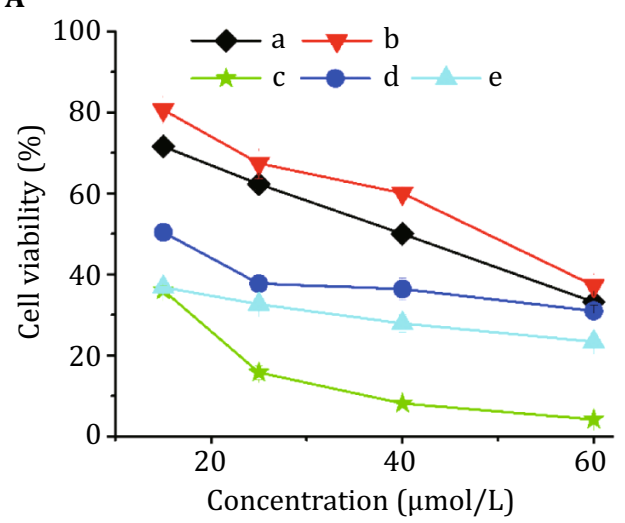

C

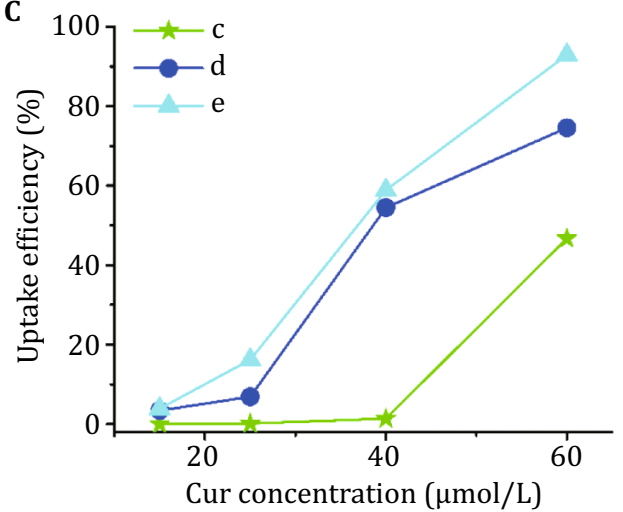

B

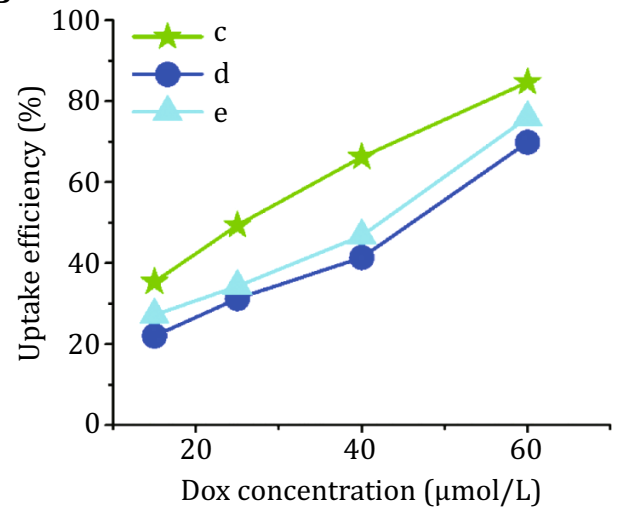

a: 24 h Cur@ANPs

b: 24 h Dox@ANPs

c: 24 h Cur/Dox@ANPs

d: 6 h Cur@ANPs, 24 h Cur@ANPs/Dox@ANPs

e: 12 h Cur@ANPs, 24h Cur@ANPs/Dox@ANPs

Fig. 3 Viability and uptake of Dox and Cur for MCF-7 breast cancer cells either treated with Cur@ANPs, Dox@ANPs, or Cur/Dox@ANPs for 24 h or pre-treated with Cur@ANPs for 6 or 12 h and then exposed to Dox@ANPs for 24 h. A Viability of NP-treated cells in function of the concentration of Cur and Dox loaded in the NPs. B Efficiency of Dox accumulation into NP-treated cells in function of the concentration of Dox loaded in the NPs. C Efficiency of Cur accumulation into NP-treated cells in function of the concentration of Cur loaded in the NPs

arising from both the drugs, suggesting the rupture of the lysosomes and the release of the drugs in the cytosol (Fig. 4C). Taken together, these results showed that, although the pre-treatment with Cur@ANPs led to a greater accumulation of Cur in the cells, Cur aggregated and remained entrapped in the lysosomes as soon as it was released from the NPs even after cell exposure to Dox@ANPs. The measurement of the lysosomal pH showed that the cells pre-treated with Cur@ANPs had a lysosomal $\mathrm{pH}$ lower than that of the cells exposed to Cur/Dox@ANPs (Fig. 5C). Since Dox can increase the lysosomal pH ( $\mathrm{Li}$ et al. 2016), we reasoned that the ability of Dox to increase the $\mathrm{pH}$ of lysosomes and trigger the accumulation of drugs in the cytosol was dependent on the aggregation state of Cur after its release from the NPs. In order to validate this hypothesis, we performed the following experiment. We added either Cur or Dox or a mixture of both the drugs in water, gently spun it down after 0 and $6 \mathrm{~h}$, and measured both the $\mathrm{pH}$ and the absorbance spectra of the supernatants. In a separate experiment, Cur was incubated in water for $3 \mathrm{~h}$ and either gently spun down and both the $\mathrm{pH}$ and the absorbance spectrum of the supernatant were measured, or free Dox was added to the mixture for an additional $3 \mathrm{~h}$. We found that when Cur and Dox were co-incubated in water, the $\mathrm{pH}$ slightly raised from 6.5 to 6.8 at $0 \mathrm{~h}$ and decreased to 6.5 after $6 \mathrm{~h}$ of incubation without forming any visible aggregates (black curves in Fig. 5A, B). The addition of Cur to water led to a decrease in the pH from 6.5 to 5.7 at $0 \mathrm{~h}$ (red curve in Fig. 5A). After $3 \mathrm{~h}$ of incubation, the $\mathrm{pH}$ further decreased to 5.5 and visible aggregates formed and were spun down after gentle centrifugation leaving a clear supernatant (green curve in Fig. 5B). When Dox was added to the Cur solution after $3 \mathrm{~h}$ of incubation, the $\mathrm{pH}$ increased to 6.1, increasing the solubility of Cur (red curve in Fig. 5B). However, only approximately half of the initial amount of Cur remained in solution in a non-aggregated form at the end of the 6-h experiment as suggested by the intensity of the peak in the Vis-range of wavelengths in the absorbance spectrum of the supernatant collected after gentle centrifugation of the 

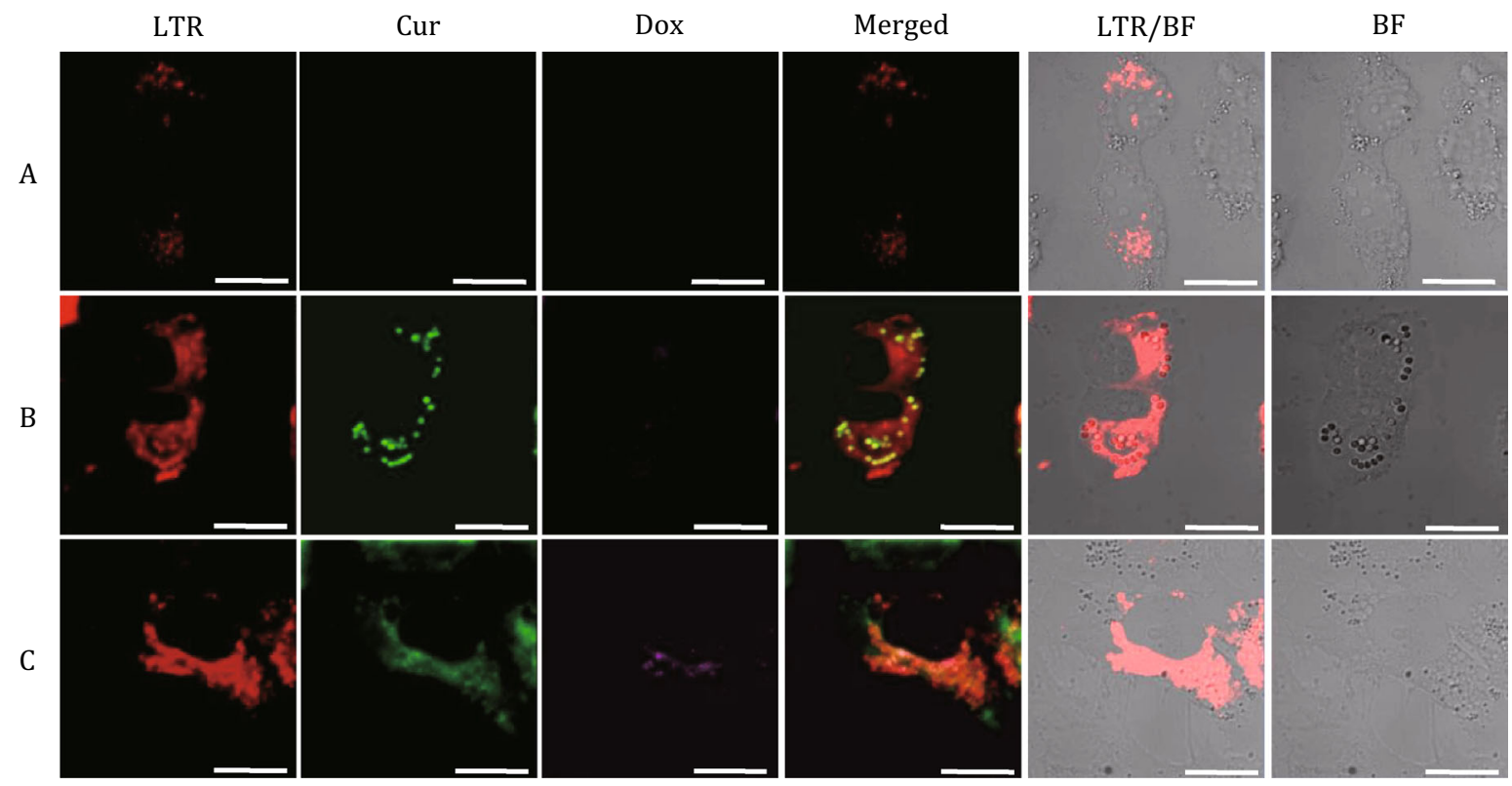

Fig. 4 Confocal laser scanning microscopy images of MCF-7 R breast cancer cells untreated (A), pre-treated with Cur@ANPs for 1.5 h and then exposed to Dox@ANPs for an additional 1.5 h (B), and treated with Cur/Dox@ANPs for 3 h (C). Scale bars are $2 \mu \mathrm{m}$ in all the images. LTR: lysotracker red, BF: bright field

mixture with respect to the intensity of the peak in the spectrum of the supernatant collected after gentle centrifugation of the solution prepared by co-incubating Cur and Dox (red curve vs. black curve in Fig. 5B).

Finally, we measured the expression of P-gp in cells treated with Cur/Dox@ANPs for $24 \mathrm{~h}$ and in cells pretreated with Cur@ANPs for $6 \mathrm{~h}$ and then exposed to Dox@ANPs for $24 \mathrm{~h}$. We found that the cells treated with Cur/Dox@ANPs exhibited a lower expression of P-gp with respect to cells pre-treated with Cur@ANPs, which suggested that Cur was able to inhibit the expression of P-gp only when it was not entrapped in the lysosomes in an aggregated form (Fig. 5D).

\section{CONCLUSIONS}

Taken together our results suggest that the synergism between Cur and Dox co-encapsulated in ANPs enabled to decrease the cell's ATT against Dox. Indeed, the simultaneous release of Cur and Dox in the lysosomes from Cur/Dox@ANPs increased the lysosomal pH and maintained Cur individually dispersed. Concomitantly, the Dox-triggered buffering of the lysosomal acidic $\mathrm{pH}$ led to the swelling of the vesicles and the release of the drugs in the cytosol. Once in the cytosol, Cur blocked the transport of Dox out of the cells by inhibiting the expression of P-gp. This ultimately translated into efficient cell killing. On the contrary, due to its weak-base nature, Cur formed aggregates in the lysosomes as soon as it was released from Cur@ANPs. Aggregated Cur decreased the buffering ability of the Dox released from Dox@ANPs added to the cell culture after the pretreatment with Cur@ANPs. This translated into less efficient release of the drugs from the lysosomes and poor inhibition of P-gp expression and, in turn, led to the transport of Dox out of the cancer cells though the P-gp pumps (Scheme 1). In conclusion, this study showed the importance of tuning the manner of co-administration of chemo-drugs and chemo-sensitizers in order to maximize the anti-cancer effects.

\section{EXPERIMENTAL DETAILS}

\section{Materials}

Cur was purchased from J\&K Co. (Beijing, China). Doxorubicin hydrochloride (Dox-HCl) was obtained from Beijing Huafeng United Technology Co., Ltd (Beijing, China). RPMI 1640 and fetal bovine serum were obtained from Wisent Inc. (Multicell, Wisent Inc., St. Bruno, Quebec, Canada). Trypsin-EDTA $(0.25 \%)$ and antibiotic solution (penicillin and streptomycin) were purchased from Invitrogen (Thermo Fisher Scientific Inc., Waltham, MA). The human breast cancer cell line MCF-7 was purchased from American Type Culture Collection (ATCC, Manassas, VA). Other chemical reagents and solvents were obtained from Alfa Aesar (Thermo Fisher Scientific). 

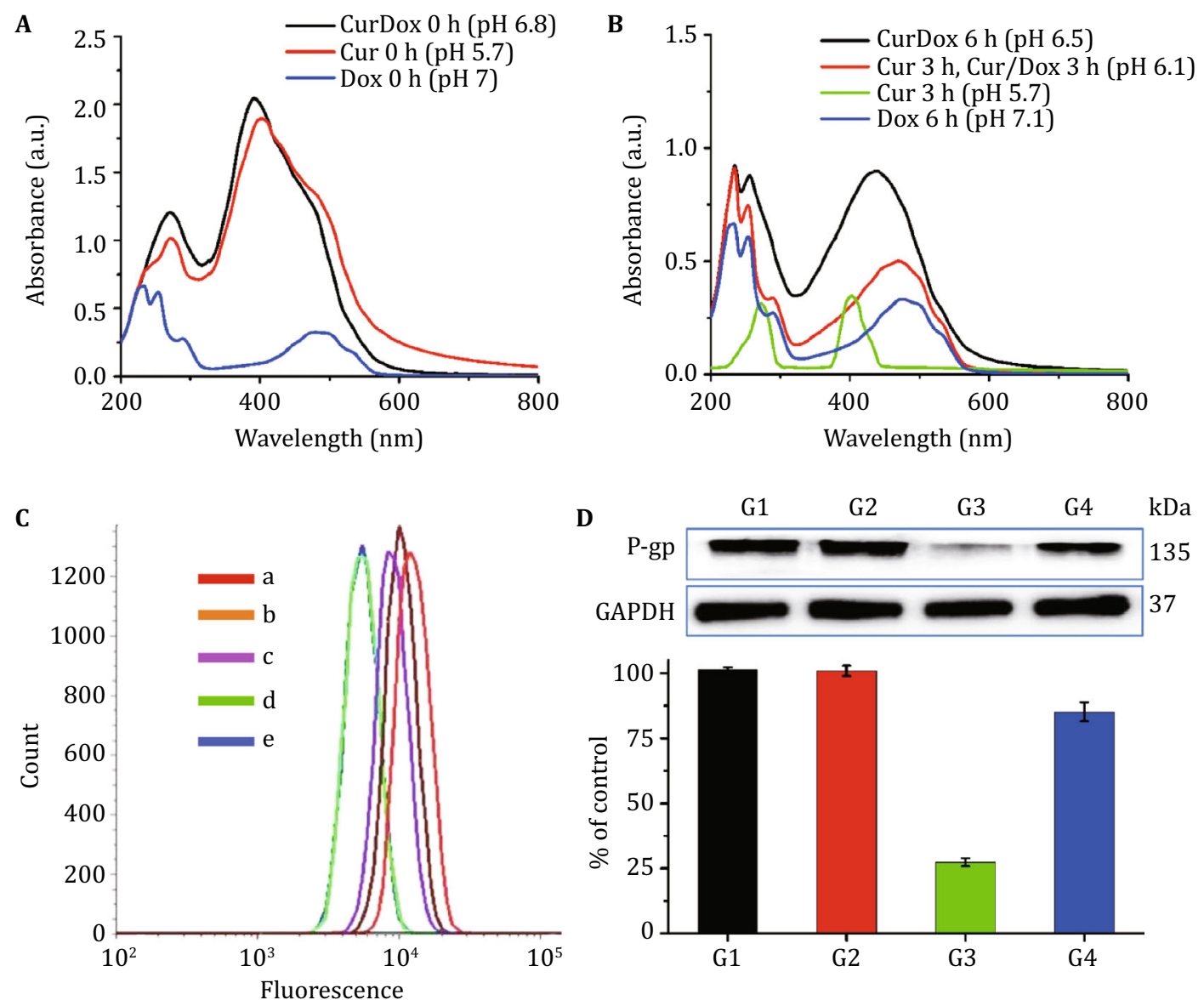
a: Control
b:12 h Cur@ANPs
c: 12 h Cur@ANPs, 24 h Cur@ANPs/Dox@ANPs
d: 24 h Cur/Dox@ANPs
e: 24 h Dox@ANPs

\author{
G1: Control \\ G2: BSA \\ G3: 24 h Cur/Dox@ANPs \\ G4: 6 h Cur@ANPs, 24hCur@ANPs/Dox@ANPs
}

Fig. 5 A, B UV-Vis absorption spectra of the supernatants collected after gentle centrifugation of the solutions prepared by incubating Cur in water for 0 and $3 \mathrm{~h}$ (red curve in $\mathbf{A}$ and green curve in B), Dox in water for 0 and $6 \mathrm{~h}$ (blue curves), a mixture of Cur and Dox in water for 0 and $6 \mathrm{~h}$ (black curves), and Dox for $3 \mathrm{~h}$ in a solution prepared by incubating Cur in water for $3 \mathrm{~h}$ (red curve in B). C LysoSensor Green DND-189 fluorescence signals measured by FACS and used as lysosomal pH probe for cells treated with vehicle (control), Cur@ANPs for 12 h, Cur@ANPs for 12 h and then treated with Dox@ANPs, Cur/Dox@ANPs for 24 h, and Dox@ANPs for 24 h. D Western blot analysis of the expression of the $135 \mathrm{kDa}$ P-glycoprotein in cells treated with vehicle (control), BSA, Cur/Dox@ANPs for 24 h, and Cur@ANPs for $12 \mathrm{~h}$ and then treated with Dox@ANPs

\section{Preparation of Cur@ANPs, Dox@ANPs, and Cur/ Dox@ANPs}

First, Dox-HCl was dissolved in DMSO at a final concentration of $15 \mathrm{mg} / \mathrm{mL}$, and then $80 \mu \mathrm{L}$ of hydrazine $(0.1 \%)$ was added to $100 \mu \mathrm{L}$ of Dox solution. The mixture was shaken for 2 min. Next, Cur was dissolved in DMSO at a final concentration of $8.3 \mathrm{mg} / \mathrm{mL}$ and the mixture was shaken for $30 \mathrm{~s}$. The Dox solution was added to an aliquot of Cur solution and the mixture was shaken for $30 \mathrm{~s}$. A BSA solution was prepared in double distilled $\mathrm{H}_{2} \mathrm{O}\left(\mathrm{ddH}_{2} \mathrm{O}\right)$ and mixed with the Cur, Dox, or Dox/Cur solution to fabricate Cur@ANPs, Dox@ANPs, and Cur/Dox@ANPs, respectively (Table 2). Glutaraldehyde was added to all the mixtures. After $3 \mathrm{~h}$ of continuous stirring at room temperature, the solutions were washed with $\mathrm{ddH}_{2} \mathrm{O}$ three times through centrifugal filter devices (100 kDa cut-off, MilliporeSigma, Burlington, MA) and the collected NPs were lyophilized and stored in the dark at $4{ }^{\circ} \mathrm{C}$. 

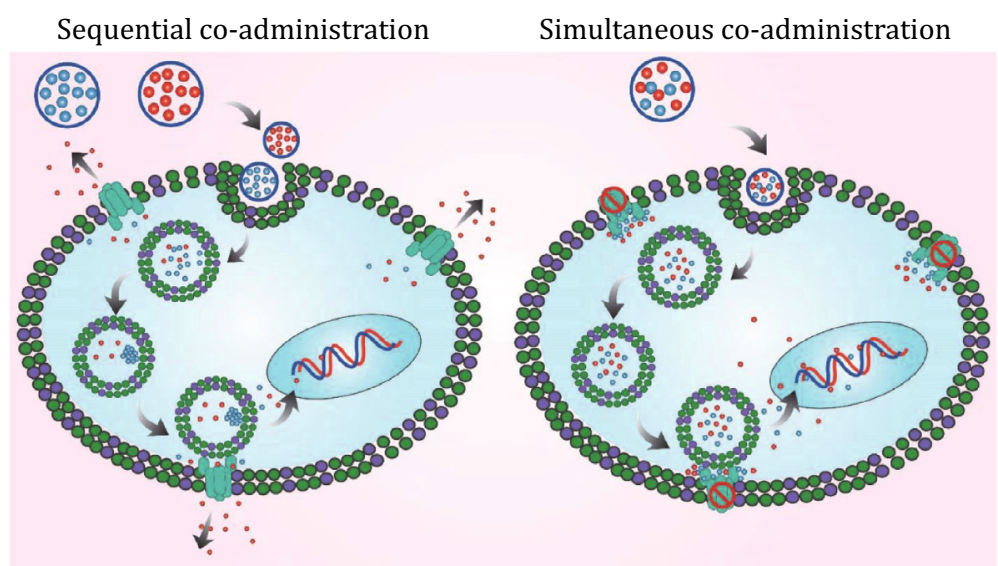

Scheme 1 This study investigated the co-administration of the chemo-sensitizer Cur and the chemo-drug Dox to the MCF-7 breast cancer cell line to block the ATT and elicit efficient cell killing. The drugs were entrapped into ANPs and co-administered to cells both sequentially and simultaneously. Sequential drug co-administration was carried out by pre-treating the cells with Cur@ANPs followed by treatment with Dox@ANPs. Simultaneous drug co-administration was carried out by treating the cells with Cur/Dox@ANPs. The simultaneous drug co-administration elicited strong cytotoxicity, whereas the sequential drug co-administration did not. The data described in this study unraveled the molecular mechanisms driving the observed different therapeutic outcomes as summarized in "Conclusions"

\section{Characterization of Cur@ANPs, Dox@ANPs, and Cur/Dox@ANPs}

The NPs' size distribution and zeta potential were calculated by a Zetasizer Nano ZS (Malvern Instruments, Worcestershire, UK). The samples were stained by uranyl acetate (1 wt\%) and the NPs' morphology was characterized by transmission electron microscopy (TEM, model JEM-200CX, JEOL, Tokyo, Japan). The $E E \%$ and $L Y \%$ of Cur and Dox in Cur@ANPs, Dox@ANPs and Cur/Dox@ANPs were measured by high-pressure liquid chromatography (HPLC, model e2796, Waters, Milford, MA) method using a reverse-phase C-18 Agilent Eclipse Plus (Thermo Fisher Scientific). The mobile phase consisted in $3 \mathrm{mmol} / \mathrm{L}$ monopotassium phosphate-acetic acid in $\mathrm{MeOH}$ (Ma et al. 2017; Zhao et al. 2015). The lyophilized NPs were dissolved in $\mathrm{MeOH}$, sonicated for $10 \mathrm{~min}$, and filtered through centrifugal filter devices (30 kDa cut-off, MilliporeSigma). The EE\% and $L Y \%$ were calculated by the following equations:

$$
E E \%=\frac{\text { The amount of loaded DOX or CUR }}{\begin{array}{l}
\text { Total amount of DOX or CUR used for ANPs } \\
\times 100,
\end{array}}
$$

$L Y \%=\frac{\text { The amount of loaded Dox or Cur }}{\text { Total amount of ANPs }} \times 100$.

DSC was performed by a diamond differential scanning calorimeter (model Diamond DSC, PerkinElmer Inc., Waltham, MA, USA). Samples were heated from 30 to $230{ }^{\circ} \mathrm{C}$ at a scan speed of $5{ }^{\circ} \mathrm{C} / \mathrm{min}$ (Jenita et al. 2014). The NPs' chemical structure was assessed by Fourier-transform infrared spectroscopy (FTIR, model IFS 66 s/v, Bruker, Ettlingen, Germany) using KBr discs. The absorption spectra of free drugs and NPs were recorded by a visible spectrophotometer (model Lambda 950, PerkinElmer), whereas their fluorescent spectra were recorded by a fluorescent spectrophotometer (model F-4600, Hitachi, Tokyo, Japan) at

Table 2 Amounts of reagents used to fabricate Cur@ANPs, Dox@ANPs, and Cur/Dox@ANPs

\begin{tabular}{lllllc}
\hline Nanoparticle & BSA $(\%)$ & Cur $(\mu \mathrm{L})$ & Dox $(\mu \mathrm{L})$ & Glutaraldehyde $(\mu \mathrm{L})$ & Time $(\mathrm{h})$ \\
\hline Cur/Dox@ANPs & 0.25 & 114.6 & 100 & 100 & 3 \\
Cur@ANPs & 1 & 114.6 & 0 & 100 & 3 \\
Dox@ANPs & 1 & 0 & 100 & 100 & 3 \\
\hline
\end{tabular}


excitation/emission (ex/em) wavelengths to detect Cur $(405 \mathrm{~nm} / 520 \mathrm{~nm})$ and Dox $(480 \mathrm{~nm} / 615 \mathrm{~nm})$.

\section{In vitro experiments}

In vitro experiments were carried out using the MCF-7 cancer cells treated with NPs both sequentially and simultaneously. Sequential drug co-administration was carried out by pre-treating the cells with Cur@ANPs loaded with $15,25,40$, or $60 \mu \mathrm{mol} / \mathrm{L}$ of Cur for 6 or $12 \mathrm{~h}$ followed by treatment with Dox@ANPs loaded with a concentration of Dox equal to that of Cur in Cur@ANPs for an additional $24 \mathrm{~h}$. Simultaneous drug co-administration was carried out by treating the cells with Cur/Dox@ANPs loaded with 15, 25, 40, or $60 \mu \mathrm{mol} / \mathrm{L}$ of Cur and Dox for $24 \mathrm{~h}$. Control cells were treated with Cur@ANPs or Dox@ANPs loaded with 15, 25, 40, or $60 \mu \mathrm{mol} / \mathrm{L}$ of Cur or Dox, respectively, for $24 \mathrm{~h}$.

The MTT assay was carried out by seeding the cells at a concentration of $5 \times 10^{3}$ cells/well in 96-well plates and growing the cells in RPMI 1640 (FBS 10\%) media at $37{ }^{\circ} \mathrm{C}, 5 \% \mathrm{CO}_{2}$ for $24 \mathrm{~h}$. The cells were treated with the NPs both sequentially and simultaneously as described before. The media was replaced with $100 \mu \mathrm{L}$ of $0.5 \mathrm{mg} / \mathrm{mL}$ MTT solution and after $4 \mathrm{~h}$ the MTT solution was replaced with $200 \mu \mathrm{L}$ of DMSO. The absorbance value was recorded at $570 \mathrm{~nm}$ with a reference wavelength of $630 \mathrm{~nm}$ using an Infinite M200 microplate reader (Tecan Group Ltd., Männedorf, Switzerland).

FACS measurements were carried out by seeding the cells in 12-well plates (three wells for each treatment) at a concentration of $3 \times 10^{5}$ cells/well and growing the cells in RPMI 1640 (FBS $10 \%$ ) media at $37{ }^{\circ} \mathrm{C}, 5 \%$ $\mathrm{CO}_{2}$ for $24 \mathrm{~h}$. The cells were treated with the NPs both sequentially and simultaneously as described before. The cells were trypsinized and washed three times. The fluorescence signals arising from the samples were measured using an Attune ${ }^{\circledR}$ acoustic focusing cytometer (Applied Biosystems, Life Technologies, Carlsbad, CA).

The co-localization of drugs within the lysosomes was carried out by seeding the cells in $35-\mathrm{mm}$ glass bottom dishes at a density of $2 \times 10^{4}$ and growing the cells in RPMI 1640 (FBS 10\%) media at $37{ }^{\circ} \mathrm{C}, 5 \% \mathrm{CO}_{2}$ for $24 \mathrm{~h}$. The cells were treated with the NPs both sequentially and simultaneously as described before. The total drug concentration in the media was $40 \mu \mathrm{mol} / \mathrm{L}$. Cells were imaged using a confocal laser scanning microscope (LSM710, Carl Zeiss Microscopy) with excitation at $405 \mathrm{~nm}$ for Cur and $488 \mathrm{~nm}$ for Dox. Lysotracker red DND-99 was used for lysosome coloring.

The measurements of lysosomal $\mathrm{pH}$ were carried out by seeding the cells in 12-well plates at a concentration of $2 \times 10^{5}$ cells/well and administering the NPs to the cells both sequentially and simultaneously as described before. The total drug concentration in the media was $40 \mu \mathrm{mol} / \mathrm{L}$. The culture media was poured out of the wells and replaced with culture media containing LysoSensor Green DND-189 at $37^{\circ} \mathrm{C}$ for $30 \mathrm{~min}$. The cells were trypsinized and washed three times. The fluorescence signals arising from the samples were measured with ex/em wavelengths of $443 \mathrm{~nm} / 505 \mathrm{~nm}$ using an Attune ${ }^{\circledR}$ acoustic focusing cytometer (Applied Biosystems).

The expression of P-gp was assessed by Western blotting (WB). Briefly, total proteins were extracted from cells, quantified by Bradford method, and run through a $10 \%$ polyacrylamide gel (SDS-PAGE). Proteins were transferred to a nitrocellulose membrane for $2 \mathrm{~h}$ at $400 \mathrm{~mA}$. The membrane was incubated in 5\% $w / v$ non-fat dry milk in TBST for $1 \mathrm{~h}$, at room temperature. The membrane was washed with TBST three times and incubated with primary antibody for P-gp and GAPDH (diluted 1:1000) overnight. The primary antibody was removed, and the membrane was washed with TBST three times and incubated with peroxidaseconjugated goat anti-rabbit IgG (diluted 1:1000) for $2 \mathrm{~h}$. Finally, the secondary antibody was removed, and the membrane was washed with TBST three times, stained with Immobilon Western reagent (Merck Millipore, Burlington, MA), and imaged by a Gel Imaging System (Bio-Rad, Hercules, CA).

Acknowledgements The authors gratefully acknowledge that this work was financially supported by the National Natural Science Foundation of China (31430031, 51373117, and 51573128) and National Distinguished Young Scholars grant (31225009). The authors also appreciate the support by the external cooperation program of BIC, Chinese Academy of Science (121D11KYSB20130006), the "Strategic Priority Research Program" of the Chinese Academy of Sciences (XDA09030301), and the NanOArt grant of the "Mission Sustainability" of the University of Rome Tor Vergata.

\section{Compliance with Ethical Standards}

Conflict of interest Seyed Mohammad Motevalli, Ahmed Shaker Eltahan, Lu Liu, Andrea Magrini, Nicola Rosato, Weisheng Guo, Massimo Bottini, and Xing-Jie Liang declare that they have no conflict of interest.

Human and animal rights and informed consent This article does not contain any studies with human or animal subjects performed by any of the authors.

Open Access This article is distributed under the terms of the Creative Commons Attribution 4.0 International License (http:// creativecommons.org/licenses/by/4.0/), which permits unrestricted use, distribution, and reproduction in any medium, provided you give appropriate credit to the original author(s) and the source, provide a link to the Creative Commons license, and indicate if changes were made. 


\section{References}

Abbasi S, Paul A, Shao W, Prakash S (2012) Cationic albumin nanoparticles for enhanced drug delivery to treat breast cancer: preparation and in vitro assessment. J Drug Deliv 2012:686108

Azizi M, Ghourchian H, Yazdian F, Bagherifam S, Bekhradnia S, Nyström B (2017) Anti-cancerous effect of albumin coated silver nanoparticles on MDA-MB 231 human breast cancer cell line. Sci Rep 7:5178

Bronze-Uhle ES, Costa BC, Ximenes VF, Lisboa-Filho PN (2017) Synthetic nanoparticles of bovine serum albumin with entrapped salicylic acid. Nanotechnol Sci Appl 10:11-21

Burger H, den Dekker AT, Segeletz S, Boersma AW, de Bruijn P, Debiec-Rychter M, Taguchi T, Sleijfer S, Sparreboom A, Mathijssen RH, Wiemer EA (2015) Lysosomal sequestration determines intracellular imatinib levels. Mol Pharmacol 88:477-487

Carvalho FS, Burgeiro A, Garcia R, Moreno AJ, Carvalho RA, Oliveira PJ (2014) Doxorubicin-induced cardiotoxicity: from bioenergetic failure and cell death to cardiomyopathy. Med Res Rev 34:106-135

Céspedes MV, Unzueta U, Tatkiewicz W, Sánchez-Chardi A, Conchillo-Solé O, Álamo P, Xu Z, Casanova I, Corchero JL, Pesarrodona M, Cedano J, Daura X, Ratera I, Veciana J, FerrerMiralles N, Vazquez E, Villaverde A, Mangues R (2014) In vivo architectonic stability of fully de novo designed protein-only nanoparticles. ACS Nano 8:4166-4176

Cheema MA, Taboada P, Barbosa S, Juárez J, Gutiérrez-Pichel M, Siddiq M, Mosquera V (2009) Human serum albumin unfolding pathway upon drug binding: a thermodynamic and spectroscopic description. J Chem Thermodyn 41:439-447

Chen Q Liu X, Chen J, Zeng J, Cheng Z, Liu Z (2015) A selfassembled albumin-based nanoprobe for in vivo ratiometric photoacoustic pH imaging. Adv Mater 27:6820-6827

Darandale SS, Vavia PR (2013) Cyclodextrin-based nanosponges of curcumin: formulation and physicochemical characterization. J Incl Phenom Macrocycl Chem 75:315-322

Deepa K, Singha S, Panda T (2014) Doxorubicin nanoconjugates. J Nanosci Nanotechnol 14:892-904

Dreis S, Rothweiler F, Michaelis M, Cinatl J Jr, Kreuter J, Langer K (2007) Preparation, characterisation and maintenance of drug efficacy of doxorubicin-loaded human serum albumin (HSA) nanoparticles. Int J Pharm 341:207-214

Ducreux M, Malka D, Mendiboure J, Etienne PL, Texereau P, Auby D, Rougier P, Gasmi M, Castaing M, Abbas M, Michel P, Gargot D, Azzedine A, Lombard-Bohas C, Geoffroy P, Denis B, Pignon JP, Bedenne L, Bouché 0 , Fédération Francophone de Cancérologie Digestive (FFCD) 2000-05 Collaborative Group (2011) Sequential versus combination chemotherapy for the treatment of advanced colorectal cancer (FFCD 2000-05): an openlabel, randomised, phase 3 trial. Lancet Oncol 12:1032-1044

Gong Z, Liu Z, Dong X, Ding YH, Dong MQ, Tang C (2017) Protocol for analyzing protein ensemble structures from chemical cross-links using DynaXL. Biophys Rep 3:100-108

Housman G, Byler S, Heerboth S, Lapinska K, Longacre M, Snyder N, Sarkar S (2014) Drug resistance in cancer: an overview. Cancers 6:1769-1792

Hsieh YW, Huang CY, Yang SY, Peng YH, Yu CP, Chao PD, Hou YC (2014) Oral intake of curcumin markedly activated CYP 3A4: in vivo and ex vivo studies. Sci Rep 4:6587

Huang P, Li Z, Hu H, Cui D (2010) Synthesis and characterization of bovine serum albumin-conjugated copper sulfide nanocomposites. J Nanomater 2010:1-6
Jayakumar R, Nair A, Rejinold NS, Maya S, Nair SV (2012) Doxorubicin-loaded pH-responsive chitin nanogels for drug delivery to cancer cells. Carbohydr Polym 87:2352-2356

Jenita JL, Chocalingam V, Wilson B (2014) Albumin nanoparticles coated with polysorbate 80 as a novel drug carrier for the delivery of antiretroviral drug-Efavirenz. Int J Pharm Investig $4: 142-148$

Kratz F (2008) Albumin as a drug carrier: design of prodrugs, drug conjugates and nanoparticles. J Control Release 132:171-183

Kuzu OF, Toprak M, Noory MA, Robertson GP (2017) Effect of lysosomotropic molecules on cellular homeostasis. Pharmacol Res 117:177-184

Lee MJ, Ye AS, Gardino AK, Heijink AM, Sorger PK, MacBeath G, Yaffe MB (2012) Sequential application of anticancer drugs enhances cell death by rewiring apoptotic signaling networks. Cell 149:780-794

Li F-Q, Su H, Wang J, Liu JY, Zhu QG, Fei YB, Pan YH, Hu JH (2008) Preparation and characterization of sodium ferulate entrapped bovine serum albumin nanoparticles for liver targeting. Int J Pharm 349:274-282

Li H, Yan K, Shang Y, Shrestha L, Liao R, Liu F, Li P, Xu H, Xu Z, Chu PK (2015) Folate-bovine serum albumin functionalized polymeric micelles loaded with superparamagnetic iron oxide nanoparticles for tumor targeting and magnetic resonance imaging. Acta Biomater 15:117-126

Li DL, Wang ZV, Ding G, Tan W, Luo X, Criollo A, Xie M, Jiang N, May H, Kyrychenko V, Schneider JW, Gillette TG, Hill JA (2016) Doxorubicin blocks cardiomyocyte autophagic flux by inhibiting lysosome acidification. Circulation 133:1668-1687

Lin X, Xie J, Niu G, Zhang F, Gao H, Yang M, Quan Q Aronova MA, Zhang G, Lee S, Leapman R, Chen X (2011) Chimeric ferritin nanocages for multiple function loading and multimodal imaging. Nano Lett 11:814-819

Lin G, Zhu W, Yang L, Wu J, Lin B, Xu Y, Cheng Z, Xia C, Gong Q Song B, Ai H (2014) Delivery of siRNA by MRI-visible nanovehicles to overcome drug resistance in MCF-7/ADR human breast cancer cells. Biomaterials 35:9495-9507

Logan R, Kong AC, Krise JP (2014) Time-dependent effects of hydrophobic amine-containing drugs on lysosome structure and biogenesis in cultured human fibroblasts. J Pharm Sci 103:3287-3296

Lohcharoenkal W, Wang L, Chen YC, Rojanasakul Y (2014) Protein nanoparticles as drug delivery carriers for cancer therapy. Biomed Res Int 2014:1-12

Lv MM, Zhu XY, Chen WX, Zhong SL, Hu Q Ma TF, Zhang J, Chen L, Tang JH, Zhao JH (2014) Exosomes mediate drug resistance transfer in MCF-7 breast cancer cells and a probable mechanism is delivery of P-glycoprotein. Tumor Biol 35:10773-10779

Ma X, Sun X, Hargrove D, Chen J, Song D, Dong Q, Lu X, Fan TH, Fu Y, Lei Y (2016) A biocompatible and biodegradable protein hydrogel with green and red autofluorescence: preparation, characterization and in vivo biodegradation tracking and modeling. Sci Rep 6:19370

Ma W, Guo Q Li Y, Wang X, Wang J, Tu P (2017) Co-assembly of doxorubicin and curcumin targeted micelles for synergistic delivery and improving anti-tumor efficacy. Eur J Pharm Biopharm 112:209-223

Mohanty C, Sahoo SK (2010) The in vitro stability and in vivo pharmacokinetics of curcumin prepared as an aqueous nanoparticulate formulation. Biomaterials 31:6597-6611

Neek M, Tucker JA, Il Kim T, Molino NM, Nelson EL, Wang SW (2018) Co-delivery of human cancer-testis antigens with adjuvant in protein nanoparticles induces higher cellmediated immune responses. Biomaterials 156:194-203 
Neerati P, Sudhakar YA, Kanwar JR (2013) Curcumin regulates colon cancer by inhibiting P-glycoprotein in in situ cancerous colon perfusion rat model. J Cancer Sci Ther 5:313

Neville-Webbe HL, Rostami-Hodjegan A, Evans CA, Coleman RE, Holen I (2005) Sequence-and schedule-dependent enhancement of zoledronic acid induced apoptosis by doxorubicin in breast and prostate cancer cells. Int J Cancer 113:364-371

Peng Y, Zhao S, Wu Y, Cao H, Xu Y, Liu X, Shui W, Cheng J, Zhao S, Shen L, Ma J, Quinn RJ, Stevens RC, Zhong G, Liu ZJ (2018) Identification of natural products as novel ligands for the human 5-HT2C receptor. Biophys Rep 4:50-61

Sahu BP, Hazarika H, Bharadwaj R, Loying P, Baishya R, Dash S, Das MK (2016) Curcumin-docetaxel co-loaded nanosuspension for enhanced anti-breast cancer activity. Expert Opin Drug Deliv 13(8):1065-1074

She W, Luo K, Zhang C, Wang G, Geng Y, Li L, He B, Gu Z (2013) The potential of self-assembled, $\mathrm{pH}$-responsive nanoparticles of mPEGylated peptide dendron-doxorubicin conjugates for cancer therapy. Biomaterials 34:1613-1623

Tarragó-Trani MT, Storrie B (2007) Alternate routes for drug delivery to the cell interior: pathways to the Golgi apparatus and endoplasmic reticulum. Adv Drug Deliv Rev 59:782-797

Umsumarng S, Pitchakarn P, Sastraruji K, Yodkeeree S, Ung AT, Pyne SG, Limtrakul P (2015) Reversal of human multi-drug resistance leukaemic cells by stemofoline derivatives via inhibition of p-glycoprotein function. Basic Clin Pharmacol Toxicol 116:390-397

Unsoy G, Khodadust R, Yalcin S, Mutlu P, Gunduz U (2014) Synthesis of doxorubicin loaded magnetic chitosan nanoparticles for $\mathrm{pH}$ responsive targeted drug delivery. Eur J Pharm Sci 62:243-250

Vinod BS, Antony J, Nair HH, Puliyappadamba VT, Saikia M, Narayanan SS, Bevin A, Anto RJ (2013) Mechanistic evaluation of the signaling events regulating curcumin-mediated chemosensitization of breast cancer cells to 5-fluorouracil. Cell Death Dis 4:e505
Wang C, Xu H, Liang C, Liu Y, Li Z, Yang G, Cheng L, Li Y, Liu Z (2013a) Iron oxide@ polypyrrole nanoparticles as a multifunctional drug carrier for remotely controlled cancer therapy with synergistic antitumor effect. ACS Nano 7:6782-6795

Wang P, Zhang L, Peng H, Li Y, Xiong J, Xu Z (2013b) The formulation and delivery of curcumin with solid lipid nanoparticles for the treatment of on non-small cell lung cancer both in vitro and in vivo. Mater Sci Eng C 33:4802-4808

Wang J, Wang F, Li F, Zhang W, Shen Y, Zhou D, Guo S (2016) A multifunctional poly (curcumin) nanomedicine for dualmodal targeted delivery, intracellular responsive release, dual-drug treatment and imaging of multidrug resistant cancer cells. J Mater Chem B 4:2954-2962

Yallapu MM, Jaggi M, Chauhan SC (2010) $\beta$-Cyclodextrin-curcumin self-assembly enhances curcumin delivery in prostate cancer cells. Colloids Surf B 79:113-125

Yan T, Li D, Li J, Cheng F, Cheng J, Huang Y, He J (2016) Effective codelivery of doxorubicin and curcumin using a glycyrrhetinic acid-modified chitosan-cystamine-poly ( $\varepsilon$-caprolactone) copolymer micelle for combination cancer chemotherapy. Colloids Surfaces B 145:526-538

Zhang Y, Yang C, Wang W, Liu J, Liu Q, Huang F, Chu L, Gao H, Li C, Kong D, Liu Q Liu J (2016) Co-delivery of doxorubicin and curcumin by $\mathrm{pH}$-sensitive prodrug nanoparticle for combination therapy of cancer. Sci Rep 6:21225

Zhao X, Chen Q Liu W, Li Y, Tang H, Liu X, Yang X (2015) Codelivery of doxorubicin and curcumin with lipid nanoparticles results in improved efficacy of chemotherapy in liver cancer. Int J Nanomed 10:257-270

Zhitomirsky B, Assaraf YG (2016) Lysosomes as mediators of drug resistance in cancer. Drug Resist Updat 24:23-33

Zhou T, Du Y, Wei T (2015) Transcriptomic analysis of human breast cancer cells reveals differentially expressed genes and related cellular functions and pathways in response to gold nanorods. Biophys Rep 1:106-114 